\title{
More frequent detection of calcified carotid atherosclerotic plaques and mineralized laryngeal cartilages on digital than on film-based panoramic radiographs
}

\author{
Sergio Lins de-Azevedo-Vaz $\mathbb{D}^{1,2, *}$, João Victor Pereira Machado $\mathbb{D}^{1}$, Teresa Cristina Rangel Pereira $\mathbb{D}^{1}$, \\ Deborah Queiroz Freitas $\mathbb{D}^{2}$ \\ ${ }^{1}$ Department of Clinical Dentistry, Federal University of Espirito Santo, Vitoria-ES, Brazil \\ ${ }^{2}$ Division of Oral Radiology, Department of Oral Diagnosis, Piracicaba Dental School, University of Campinas, Piracicaba-SP, Brazil
}

\section{ABSTRACT}

\begin{abstract}
Purpose: To determine whether calcified carotid atherosclerotic plaques (CCAPs) and mineralized laryngeal cartilages (MLCs) were more frequently detected on digital or film-based panoramic radiographs. The clinical relevance of this question is that some radiopacities seen on digital radiographs may correspond to medium-density tissues that are not necessarily mineralized.

Materials and Methods: Data were collected from panoramic radiographs and the respective reports issued by 2 private oral radiology centers. A total of 388 radiographs and reports were divided into film-based (group A) and digital (group D) radiographs. The frequencies of CCAPs and MLCs were analyzed using the Fisher exact test, and odds ratios were also calculated $(\alpha=1 \%)$.

Results: The mean age of patients whose reports and radiographs showed CCAPs and/or MLCs ranged from 50.1 to 54.1 years. There was a predominance of females. A higher frequency of CCAPs and MLCs was observed in group D than in group A at both centers $(P<0.01)$. CCAPs and MLCs were detected 4 times more frequently in group D than in group A at one of the centers.

Conclusion: CCAPs and MLCs were more frequently detected on digital than on film-based panoramic radiographs. Further studies are needed to determine whether such radiopacities do indeed correspond to mineralized, rather than medium-density, tissues.(Imaging Sci Dent 2019; 49: 65-70)
\end{abstract}

KEY WORDS: Atherosclerosis; Incidental Findings; Radiography, Panoramic; Radiography, Dental, Digital

\section{Introduction}

Panoramic radiography is used for many clinical purposes in dentistry, since it permits visualization of the upper and lower teeth, maxilla, mandible, and adjacent anatomical structures, such as the maxillary sinuses and hyoid bone. Radiopacities suggestive of soft tissue mineralization in adjacent anatomical structures are seen on $4 \%-19 \%$ of panoramic radiographs, and include salivary stones, mineralized lymph nodes, tonsiloliths, phleboliths, calcified

*We are thankful to the National Council for Scientific and Technological Development in Brazil for a scholarship grant.

Received August 24, 2018; Revised November 10, 2018; Accepted November 23, 2018

*Correspondence to : Prof. Sergio Lins de-Azevedo-Vaz

Departamento de Clínica Odontológica, Av. Marechal Campos, 1468, Maruípe,

Vitória-ES, Brasil 29.047-105

Tel) 55-27-3335-7242, E-mail) sergio.vaz@ufes.br carotid atherosclerotic plaques (CCAPs), and mineralized laryngeal cartilages (MLCs). ${ }^{1-3}$ The differential diagnosis is important because some of these conditions have a similar appearance, but only few require immediate intervention. ${ }^{4}$

CCAPs are caused by endothelial injury and appear as irregular and heterogeneous radiopaque images on panoramic radiographs, projected between the third and fourth cervical vertebrae inferior to the mandibular angle. ${ }^{4}$ CCAPs can cause ischemic stroke, and more commonly affect older male patients. ${ }^{5}$ Other risk factors include diabetes, hypertension, obesity, a sedentary lifestyle, and alcohol and tobacco consumption. ${ }^{5}$ If a CCAP is suspected, the patient should be referred to a physician for further evaluation.,

The laryngeal cartilages provide physical support to the larynx, and include the arytenoid, corniculate, cuneiform, cricoid, epiglottis, and thyroid cartilage. Mineralization of

Copyright (c) 2019 by Korean Academy of Oral and Maxillofacial Radiology

This is an Open Access article distributed under the terms of the Creative Commons Attribution Non-Commercial License (http://creativecommons.org/licenses/by-nc/3.0) which permits unrestricted non-commercial use, distribution, and reproduction in any medium, provided the original work is properly cited. Imaging Science in Dentistry · pISSN 2233-7822 eISSN 2233-7830 
the superior horn of the thyroid cartilage appears as a vertical radiopacity measuring $4 \mathrm{~mm}$ in width and $15 \mathrm{~mm}$ in length, medial to the fourth cervical vertebra. ${ }^{4}$ The triticeous cartilages are other small cartilages located in the lateral thyrohyoid ligament that appear as radiopaque images measuring 2 to $4 \mathrm{~mm}$ in width and 7 to $9 \mathrm{~mm}$ in length when mineralized, also at the level of the fourth cervical vertebra. ${ }^{4}$

The radiographic appearance of CCAPs is quite similar to that of some MLCs, such as the thyroid and triticeous cartilages. Characteristics such as morphology and location may aid in the differential diagnosis, since MLCs are mostly limited to the greater hyoid horn and the thyroid cartilage, while CCAPs are usually not restricted to the hyoid level. ${ }^{7}$

As film-based radiographs are being replaced with digital radiographs, our experience has shown an increasing frequency of cervical radiopacities on digital panoramic radiographs. Studying a similar population that had been previously examined, Beckstrom et al. ${ }^{8}$ in 2007 likewise reported a higher prevalence of CCAPs on digital panoramic images than on film-based radiographs. In terms of the clinical relevance of this issue, we hypothesize that some of these radiopacities may correspond to medium-density tissues that are not necessarily mineralized, and appear to be more frequently detected on digital radiographs due to technical differences in image processing between analogue and digital technologies.

This study aimed to assess whether CCAPs and MLCs were more frequently detected on digital than on film-based radiographs using samples of 2 populations from distinct geographic regions.

\section{Materials and Methods}

This cross-sectional observational study was approved by the local Research Ethics Committee. The sample consisted of panoramic radiographs and reports obtained from 2 private oral radiology centers located in the northeast $(\mathrm{C} 1)$ and southeast (C2) regions of Brazil, which had replaced filmbased systems with digital systems 3 years before the study. Film-based radiographs were obtained using the Orthophos 3 machine (Sirona Dental Systems, Bensheim, Germany) and Kodak T-MAT 15- ×30-cm films (Eastman Kodak, Rochester, NY, USA) processed in an A/T $2000 \mathrm{XR}$ automatic film machine (Air Techniques, Melville, NY, USA) at both centers. Digital radiographs were acquired with the Kodak 8000 digital panoramic system (Eastman Kodak, Rochester, NY, USA) at C1 and with the Orthophos XG5 system (Sirona Dental Systems, Bensheim, Germany) at C2. The mean exposure parameters used for these machines are shown in Table 1.
Table 1. Mean exposure parameters for each radiographic system

\begin{tabular}{lccc}
\hline System & $\begin{array}{c}\text { Tube voltage } \\
(\mathrm{kVp})\end{array}$ & $\begin{array}{c}\text { Tube current } \\
(\mathrm{mA})\end{array}$ & $\begin{array}{c}\text { Exposure time } \\
(\mathrm{s})\end{array}$ \\
\hline Orthophos 3 & & & \\
Adult men & 74 & 10 & 11.3 \\
Adult women & 72 & 10 & 11.3 \\
Children & 64 & 10 & 11.3 \\
Orthophos XG5 & & & \\
Adult men & 68 & 8 & 14.1 \\
Adult women & 64 & 8 & 14.1 \\
Children & 62 & 8 & 14.1 \\
Kodak 8000 & & & \\
Adult men & 73 & 10 & 13.9 \\
Adult women & 71 & 10 & 13.9 \\
Children & 71 & 6.3 & 13.2 \\
\hline
\end{tabular}

Patients older than 30 years of both sexes, whose radiographs and reports were analyzed and issued by the same oral radiologist with more than 5 years of clinical experience, were included. Reports mentioning difficulties during patient positioning and radiographs with significant technical and processing errors were excluded from the study. The final sample consisted of 388 panoramic radiographs and 388 reports, corresponding to the calculated sample size. The reports and radiographs included in the study were assigned to 2 groups: film-based panoramic radiographs and their reports $(n=188$; group A), and digital panoramic radiographs and their reports $(n=200$; group $D)$.

First, all reports were analyzed by an investigator who counted the presence or absence of CCAPs and MLCs in a $2 \times 2$ table. Prior to the study, radiographs from group A were routinely digitized at $\mathrm{C} 1$ and $\mathrm{C} 2$ using an HP Scanjet G4050 scanner with a transparent materials adapter (Hewlett Packard Enterprise, San José, CA, USA) at 300 DPI resolution and saved as TIFF files.

The digitized radiographs from groups $\mathrm{A}$ and $\mathrm{D}$ were assessed on a desktop computer (Optiplex 790; Dell Inc., Round Rock, TX, USA) on a 19" monitor with a standard resolution of 1366 × 768 (E1916H; Dell Inc., Round Rock, TX, USA), using the Windows imaging viewer and the zoom tool. Two oral radiologists with 10 to 20 years of experience with panoramic radiographs assessed the images in a silent room under dim lighting conditions. Without prior knowledge of the content of the reports, the oral radiologists assessed all panoramic radiographs to ensure the consistency of the data collected in the reports. They also analyzed the radiographic features of CCAPs and MLCs and indicated their most likely diagnostic impression (i.e., whether radiopacities were suggestive of CCAP, mineralized triticeous cartilage, or mineralized superior horn of 
the thyroid cartilage) according to the criteria described by Carter et al. (2000). ${ }^{4}$ The pooled data were analyzed according to population (C1 vs. C2), group (A vs. D), side (right vs. left vs. both), sex (male vs. female), and age.

Data were tabulated in Microsoft Excel (Microsoft Corp., Redmond, WA, USA) spreadsheets and analyzed using the Fisher exact test; odds ratios were also calculated, adopting a significance level of $1 \%$. The descriptive analysis included the absolute and relative frequency, mean, and standard deviation. The null hypothesis was that CCAP/MLC detection would not differ between groups $\mathrm{A}$ and $\mathrm{D}$.

\section{Results}

Table 2 shows the age and sex of the patients in the sample. There was a predominance of female patients at both centers, and the patients' age ranged from 31 to 87 years. The mean age of groups $\mathrm{A}$ and $\mathrm{D}$ was similar at $\mathrm{C} 2$, but group A had a lower mean age and a smaller standard deviation than group $\mathrm{D}$ at $\mathrm{C} 1$.

All reports matched the opinion of the oral radiologists when the radiographs were assessed for the presence or absence of CCAPs and MLCs. Table 3 shows the frequencies of CCAPs and MLCs. At C1, CCAPs and MLCs were detected on 17 panoramic radiographs from group $\mathrm{D}$, but on none of the radiographs from group A. At C2, CCAPs and MLCs were detected on 30 radiographs from group D, but on only 9 radiographs from group A. Therefore, CCAPs and MLCs were more frequently detected on digital panoramic radiographs at both $\mathrm{C} 1$ and $\mathrm{C} 2(P<0.01)$. The odds ratio could only be calculated for $\mathrm{C} 2$ because of the absence of positive cases in group $\mathrm{A}$ at $\mathrm{C} 1$. The detection of

Table 2. Sample characteristics: age and sex

\begin{tabular}{cllcrr}
\hline Center & \multicolumn{1}{c}{ Sex $-\mathrm{n}(\%)$} & \multicolumn{1}{c}{ Group } & Age (Mean \pm SD) & Minimum age & Maximum age \\
\hline C1 $(\mathrm{n}=188)$ & Male-77 $(41.0 \%)$ & Film-based(A) & $36.7 \pm 7.8$ & 31 & 75 \\
& Female-111(59.0\%) & Digital(D) & $42.7 \pm 12.7$ & 31 & 87 \\
C2 $(\mathrm{n}=200)$ & Male-89(44.5\%) & Film-based(A) & $46.5 \pm 11.4$ & 31 & 78 \\
& Female-111(55.5\%) & Digital(D) & $44.5 \pm 12.9$ & 31 & 81 \\
\hline
\end{tabular}

$\mathrm{n}$ : absolute number, $\mathrm{C} 1$ : center 1, C2: center 2, SD: standard deviation

Table 3. Radiopacities suggesting calcified carotid atherosclerotic plaques and/or mineralized laryngeal cartilages

\begin{tabular}{|c|c|c|c|c|c|}
\hline Center & Group & Presence $-\mathrm{n}(\%)$ & Absence $-\mathrm{n}(\%)$ & $P$ value* & Odds ratio \\
\hline \multirow[t]{2}{*}{$\mathrm{C} 1(\mathrm{n}=188)$} & Film-based (A) & $0(0 \%)$ & $88(100 \%)$ & $<0.01$ & $-{ }^{\dagger}$ \\
\hline & Digital(D) & $17(17 \%)$ & $83(83 \%)$ & & \\
\hline \multirow[t]{2}{*}{$\mathrm{C} 2(\mathrm{n}=200)$} & Film-based (A) & $9(9 \%)$ & $91(91 \%)$ & $<0.01$ & $4.33^{\ddagger}$ \\
\hline & Digital(D) & $30(30 \%)$ & $70(70 \%)$ & & $1.93-9.71^{\S}$ \\
\hline
\end{tabular}

$\mathrm{n}$ : absolute number, ${ }^{*}$ According to the Fisher exact test, ${ }^{\dagger}$ Not calculated because of the absence of positive cases in the film-based group, ${ }^{\dagger}$ Statistically significant $(P<0.01),{ }^{\S} 95 \%$ confidence interval

Table 4. Characteristics of pooled positive cases according to diagnostic impression

\begin{tabular}{|c|c|c|c|c|}
\hline & & $\begin{array}{l}\text { Calcified carotid atherosclerotic plaque } \\
\qquad \mathrm{N}(\%)\end{array}$ & $\begin{array}{l}\text { Mineralized triticeous cartilage } \\
\qquad \mathrm{N}(\%)\end{array}$ & $\begin{array}{l}\text { Mineralized superior horn } \\
\text { of the thyroid cartilage } \\
\qquad N(\%)\end{array}$ \\
\hline \multirow[t]{2}{*}{ Population } & $\mathrm{C} 1$ & $4(50.0 \%)$ & $1(17.0 \%)$ & $12(29.0 \%)$ \\
\hline & $\mathrm{C} 2$ & $4(50.0 \%)$ & $5(83.0 \%)$ & $30(71.0 \%)$ \\
\hline \multirow[t]{2}{*}{ Group } & Film-based (A) & $1(12.5 \%)$ & $0(0.0 \%)$ & $8(19.0 \%)$ \\
\hline & Digital (D) & $7(87.5 \%)$ & $6(100.0 \%)$ & $34(81.0 \%)$ \\
\hline \multirow[t]{3}{*}{ Side } & Right & $5(62.5 \%)$ & $0(0.0 \%)$ & $12(28.5 \%)$ \\
\hline & Left & $2(25.0 \%)$ & $3(50.0 \%)$ & $1(2.5 \%)$ \\
\hline & Both & $1(12.5 \%)$ & $3(50.0 \%)$ & $29(69.0 \%)$ \\
\hline \multirow[t]{2}{*}{ Sex } & Male & $7(87.5 \%)$ & $2(33.0 \%)$ & $11(26.1 \%)$ \\
\hline & Female & $1(12.5 \%)$ & $4(64.0 \%)$ & $31(73.9 \%)$ \\
\hline Mean age & & $54.1 \pm 6.8$ & $53.5 \pm 15.7$ & $50.1 \pm 12.4$ \\
\hline Total & $56(100.0 \%)$ & $8(14.3 \%)$ & $6(10.7 \%)$ & $42(75.0 \%)$ \\
\hline
\end{tabular}




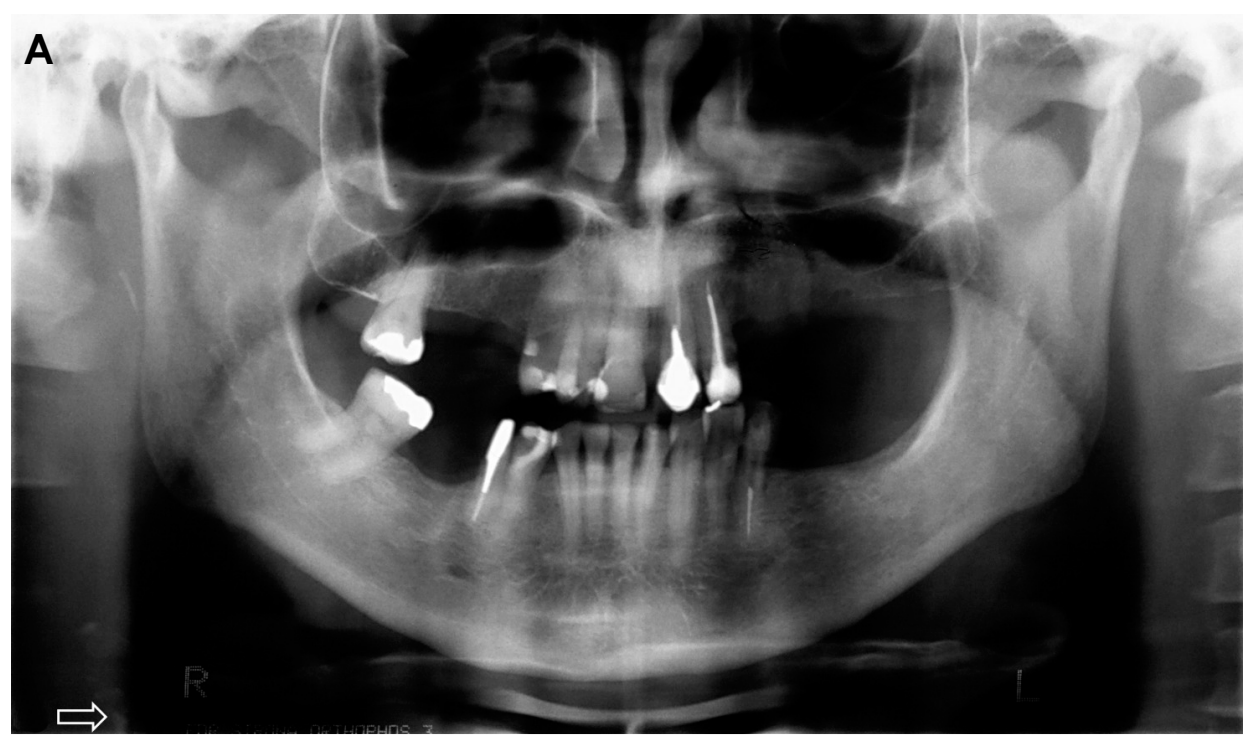

Fig. 1. Film-based (A) and digital(B) panoramic radiographs show radiopacities suggestive of mineralized laryngeal cartilages (superior horn of the thyroid cartilage) indicated by white arrows.

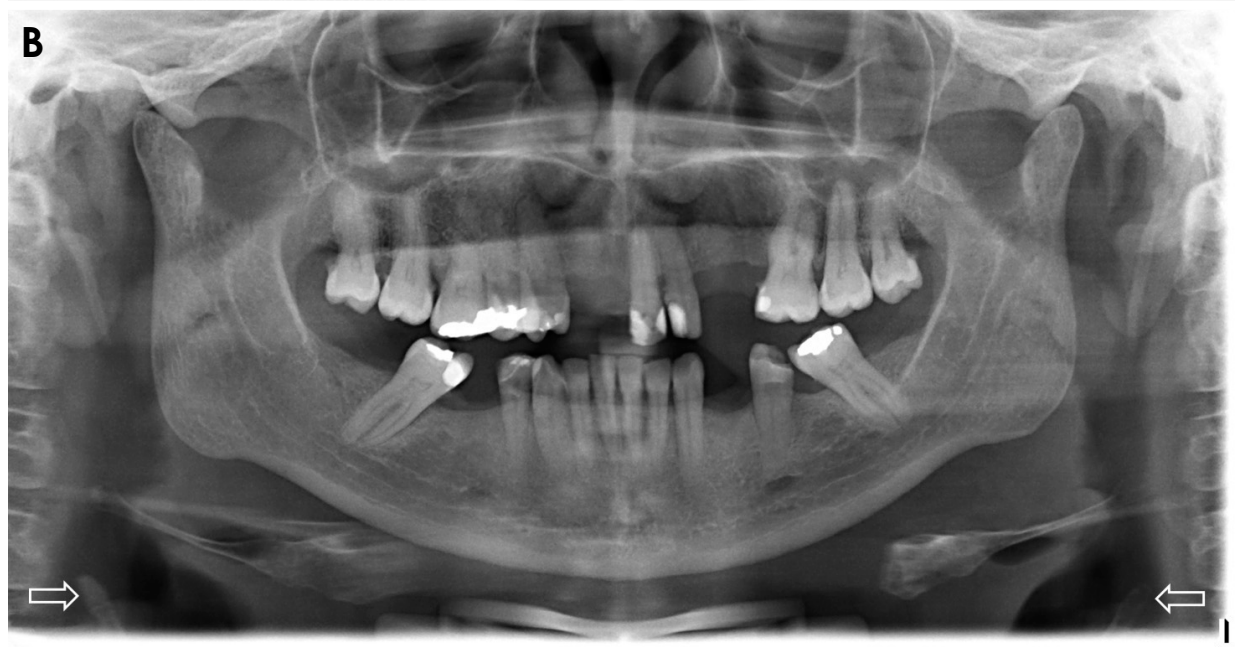

CCAPs and MLCs was 4.33 times more frequent in group $\mathrm{D}$ than in group A at C2.

Table 4 shows the pooled data of positive cases. The frequency of CCAPs was the same in both populations. CCAPs were more frequent on the right side and in male patients, and the mean age of patients with CCAPs was 54.1 years. Mineralized triticeous cartilage was more frequently detected at C2 and in female patients, and the mean age of patients with mineralized triticeous cartilage was 53.5 years. Three cases were found on the left side, and the other 3 on both sides. Mineralized superior horn of the thyroid cartilage was also more prevalent at $\mathrm{C} 2$, on both sides, and in female patients; the mean age of the affected patients was 50.1 years. The frequency of all types of radiopacities was higher in group D than in group A. Figure 1 shows examples of radiopacities suggestive of mineralized superior horn of the thyroid cartilage on film-based and digital panoramic radiographs. Radiopacities seemed to be more evi- dent on digital images than on film-based radiographs.

\section{Discussion}

Our hypothesis was confirmed, since statistically significant differences in CCAP/MLC frequencies were found between groups $\mathrm{A}$ and $\mathrm{D}$ at both centers $(P<0.01)$. A calculation of the odds ratio revealed that 4 times more cervical radiopacities were detected on digital panoramic radiographs than on film-based images. This ratio could only be calculated for $\mathrm{C} 2$, since no positive cases were found in group $\mathrm{A}$ at $\mathrm{C} 1$. Interestingly, at $\mathrm{C} 1$, the patients in group $\mathrm{A}$ were younger than those in group D. Our hypothesis arose from our daily practice in oral radiology and imaging reports, which gave us the impression that we were seeing more images consistent with CCAPs/MLCs on digital radiographs. Since no studies on the subject were available in the literature, we decided to evaluate whether our im- 
pression was correct. Two oral radiology centers located in distinct geographic regions were included in the study. Film-based panoramic radiographs were compared to digital radiographs obtained at the same center to rule out the influence of local factors on the variables studied.

CCAPs and MLCs were counted together for statistical purposes because most of the reports did not describe whether the radiopacity was a CCAP or MLC. Furthermore, the radiographic appearance of CCAPs and MLCs is very similar, and both are included in the differential diagnosis. ${ }^{9,10}$ Two oral radiologists (independent examiners) also assessed all panoramic radiographs, and the most likely diagnostic impression was considered to generate the pooled data shown in Table 4.

Considering all 56 radiopacities found in the study, 8 were suggestive of CCAP $(14.3 \%), 6$ were suggestive of mineralized triticeous cartilages (10.7\%), and 42 were suggestive of mineralized superior horn of the thyroid cartilage $(75.0 \%)$. Most patients with CCAP were male (87.5\%), in agreement with Friedlander et al. $(2005)^{5}$, but we found a lower mean age than those authors. This difference is probably due to the fact that our patients were older than 30 years, while the patients analyzed by Friedlander et al. were older than 50 years. MLCs were more prevalent in female patients (72.9\%); the mean age was 53.5 years for mineralized triticeous cartilage and 50.1 years for mineralized superior horn of the thyroid cartilage. Although the occurrence of mineralization tends to increase with age, the degree of mineralization and chronological age are not well correlated. ${ }^{4}$ In the study of Claassen et al. $(2014)^{11}$, both male and female subjects in the fourth and fifth decades of life were more likely to exhibit mineralized superior horn of the thyroid cartilage, which is in accordance with the respective mean age found in the present study. However, in our study, more female patients had mineralized superior horn of the thyroid cartilage, whereas mineralized thyroid cartilage was more frequent among men in the study of Claassen et al. (2014). ${ }^{11}$ Regarding location, most CCAPs were seen on the right side $(62.5 \%)$, while mineralized superior horn of the thyroid cartilage was found on both sides (69.0\%).

A modified anteroposterior radiograph of the skull can be used to differentiate between CCAP and MLC. ${ }^{6}$ However, due the limitations of that modality, ultrasound examination with a carotid echo-Doppler is recommended for diagnosis and treatment planning in cases of CCAP. ${ }^{5}$ In our clinical practice, we usually refer patients for a medical examination when CCAPs are seen on imaging. Conversely, since MLCs are physiological, there are no associated risk factors or clinical implications. ${ }^{7}$ Panoramic radiography, which is used for many purposes in clinical practice, may be relevant for the early diagnosis of CCAP.

One may hypothesize that the more frequent detection of calcifications in group D may have been due to lifestyle changes among the population that would increase the prevalence of CCAPs. However, this explanation cannot be accepted, because the radiographs of group A were acquired only 1 to 3 years before those of group $\mathrm{D}$, a period that is not sufficient for lifestyle changes to influence the prevalence of CCAPs. We therefore believe that the present results can be explained by a reduction in the energy parameters ( $\mathrm{mA}$ and $\mathrm{kVp}$ ) employed in digital systems. In addition, the possibility to adjust brightness and contrast in the images may enable the visualization of medium-density anatomical structures, such as cartilages. Therefore, it is possible that some of the radiopacities found on digital panoramic radiographs correspond to normal cartilages that appeared as radiopaque areas due to the reduction in energy parameters and image adjustment. We believe that medium-density tissues, which used to be burned out by the higher energy parameters applied to film-based radiographs and the low resistance offered by the cervical region, are now being seen in digital radiographs due to the factors mentioned above. Ultrasound studies are recommended to confirm this hypothesis, since ultrasonography is a noninvasive method that uses non-ionizing radiation.

The major limitations of this study are the different devices used and the small number of oral radiology centers included. It was difficult to gather a sample of film-based radiographs from centers that have recently switched to a digital system. The energy factors were also different, since they varied according to the patient's physical characteristics, but this represents actual clinical practice. However, we do not believe that the digitization of film-based panoramic radiographs influenced our results, since the opinions of the observers who evaluated the digitized images from the database were the same as those presented in the reports, which were issued by professionals that had analyzed the physical version of the film-based radiographs.

In conclusion, CCAPs and/or MLCs were more frequently detected on digital images than on film-based panoramic radiographs. Further studies are encouraged to determine whether such radiopacities do indeed correspond to mineralized structures, rather than medium-density anatomical structures such as cartilages.

\section{Acknowledgements}

The authors thank Drs. Gláucia Leão, Liliani Moro, and 
Vanessa Mansk for allowing access to the sample studied.

\section{References}

1. Monsour PA, Romaniuk K, Hutchings RD. Soft tissue calcifications in the differential diagnosis of opacities superimposed over the mandible by dental panoramic radiography. Aust Dent J 1991; 36: 94-101.

2. Bayer S, Helfgen EH, Bös C, Kraus D, Enkling N, Mues S. Prevalence of findings compatible with carotid artery calcifications on dental panoramic radiographs. Clin Oral Investig 2011; 15: 563-9.

3. Sutter W, Berger S, Meier M, Kropp A, Kielbassa AM, Turhani D. Cross-sectional study on the prevalence of carotid artery calcifications, tonsilloliths, calcified submandibular lymph nodes, sialoliths of the submandibular gland, and idiopathic osteosclerosis using digital panoramic radiography in a Lower Austrian subpopulation. Quintessence Int 2018; 49: 227-38.

4. Carter LC. Discrimination between calcified triticeous cartilage and calcified carotid atheroma on panoramic radiography. Oral Surg Oral Med Oral Pathol Oral Radiol Endod 2000; 90: 10810.

5. Friedlander AH, Garrett NR, Chin EE, Baker JD. Ultrasonographic confirmation of carotid artery atheromas diagnosed via panoramic radiography. J Am Dent Assoc 2005; 136: 635-40.

6. Brito AC, Nascimento HA, Argento R, Beline T, Ambrosano GM, Freitas DQ. Prevalence of suggestive images of carotid artery calcifications on panoramic radiographs and its relationship with predisposing factors. Cien Saude Colet 2016; 21: 2201-8.

7. Ahmad M, Madden R, Perez L. Triticeous cartilage: prevalence on panoramic radiographs and diagnostic criteria. Oral Surg Oral Med Oral Pathol Oral Radiol Endod 2005; 99: 225-30.

8. Beckstrom BW, Horsley SH, Scheetz JP, Khan Z, Silveira AM, Clark SJ, et al. Correlation between carotid area calcifications and periodontitis: a retrospective study of digital panoramic radiographic findings in pretreatment cancer patients. Oral Surg Oral Med Oral Pathol Oral Radiol Endod 2007; 103: 359-66.

9. Carter LC, Tsimidis K, Fabiano J. Carotid calcifications on panoramic radiography identify an asymptomatic male patient at risk for stroke. A case report. Oral Surg Oral Med Oral Pathol Oral Radiol Endod 1998; 85: 119-22.

10. Almog DM, Tsimidis K, Moss ME, Gottlieb RH, Carter LC. Evaluation of a training program for detection of carotid artery calcifications on panoramic radiographs. Oral Surg Oral Med Oral Pathol Oral Radiol Endod 2000; 90: 111-7.

11. Claassen H, Schicht M, Sel S, Paulsen F. Special pattern of endochondral ossification in human laryngeal cartilages: X-ray and light-microscopic studies on thyroid cartilage. Clin Anat 2014; 27: 423-30. 